Modern Physics Letters A

(C) World Scientific Publishing Company

\title{
LEPTON FLAVOR VIOLATION DECAYS OF VECTOR MESONS IN UNPARTICLE PHYSICS
}

\author{
$\mathrm{KE}-\mathrm{SHENG}$ SUN $^{\dagger, \ddagger, *}$, TAI-FU FENG ${ }^{\ddagger}, \dagger, \mathrm{LI}_{-N A} \mathrm{KOU}^{\dagger}$, FEI SUN ${ }^{\dagger}$, \\ TIE-JUN GAO $\stackrel{\ddagger}{\dagger}$, HAI-BIN ZHANG ${ }^{\ddagger}, \dagger$ \\ $\dagger$ Department of Physics, Dalian University of Technology, Dalian 116024, China \\ $\ddagger$ Department of Physics, Hebei University, Baoding 071002, China \\ *sunkesheng@126.com
}

Received (Day Month Year)

Revised (Day Month Year)

\begin{abstract}
We investigate the lepton flavor violation decays of vector mesons in the scenario of the unparticle physics by considering the constraint from $\mu-e$ conversion. In unparticle physics, the predictions of LFV decays of vector mesons depend strongly on the scale dimension $d_{\mathcal{U}}$. The predictions of LFV decays of vector mesons can reach the detective sensitivity in experiment in region of $3 \leq d_{\mathcal{U}} \leq 4$, while the prediction of $\mu-e$ conversion rate can meet the experimental upper limit. For the searching of the lepton flavor violation processes of charged lepton sector in experiment, the process $\Upsilon \rightarrow e \mu$ may be a promising one to be observed.
\end{abstract}

Keywords: Lepton flavor violating; Unparticle.

PACS Nos.:13.20.-v, 12.60.-i

\section{Introduction}

During the last decades, searching for Lepton Flavor Violation (LFV) processes in charged lepton sector, as an evidence to discover new physics beyond the Standard Model (SM), have attracted a great deal of attention. Although nonzero neutrino masses supported by the neutrino oscillation experiments $\frac{11213}{1}$ imply the nonconservation of lepton flavor, due to the small masses of neutrinos, the lepton flavor violating processes in the SM are highly suppressed. Nevertheless, the LFV decays could be enhanced by the new sources of LFV in various extensions of the SM, such as grand unified models, ${ }^{4 / 5 \mid 6}$ supersymmetric models with and without Rparity, $7 / 899$ left-right symmetry models etc! $10|11| 12$ These new sources are mainly originated from the interactions between the SM particles and new particles beyond SM. Instead, Georgi proposes an alternative scenario that there could be a sector that is exactly scale invariant and very weakly interacting with the sectors in SM $13 \mid 14$ There are no particles in such a sector in space-times spaces cause no particles states with a nonzero mass exist. In general, the scale invariant sector or the so-called unparticle has a scale dimension of fractional number rather than an 
integral number. The interactions between the unparticle and the SM particles in low energy effective theory can lead to various interesting features in LFV processes and other phenomenologies. In unparticle physics, the unparticle can interact with different flavors of SM leptons and this indicates that the LFV processes can happen at tree level. There have been many studies of LFV processes in unparticle physics.

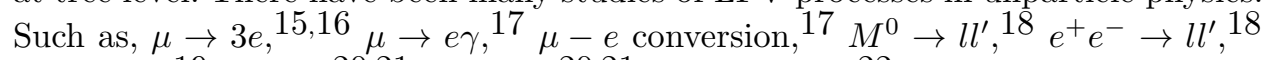
$\left.\left.\left.J / \Psi \rightarrow l l^{\prime}, 19\right] r l l^{\prime}, 20 \mid 21\right] \rightarrow l^{\prime} \gamma \gamma, 20 \mid 21\right] \tau \rightarrow l\left(V_{0}, P_{0}\right), 22$ etc..

The study of LFV processes involving vector mesons is an effective way maybe to search for new physics beyond the SM, and the SND Collaboration at the BINP (Novosibirk) presents an upper limit on the $\phi \rightarrow e^{+} \mu^{-}$branching fraction of $\operatorname{BR}\left(\phi \rightarrow e^{+} \mu^{-}\right) \leq 2 \times 10^{-6}\left[23\right.$ Additionally, using a sample of $5.8 \times 10^{7} \mathrm{~J} / \Psi$ events collected with the BESII detector, Ref. 24 obtains the upper limits on $\operatorname{BR}(J / \Psi \rightarrow \mu \tau)<2.0 \times 10^{-6}$ and $\operatorname{BR}(\Upsilon \rightarrow \mu \tau)<8.3 \times 10^{-6}$ at the $90 \%$ confidence level (C.L.). Adopting the data collected with the CLEO III detector, the authors of Ref. 25 estimate the upper limits on $\operatorname{BR}(\Upsilon(1 S) \rightarrow \mu \tau)<6.0 \times 10^{-6}$, $\mathrm{BR}(\Upsilon(2 S) \rightarrow \mu \tau)<1.4 \times 10^{-5}$ and $\mathrm{BR}(\Upsilon(3 S) \rightarrow \mu \tau)<2.0 \times 10^{-5}$ respectively at the $95 \%$ C.L. In literatures, several stringent limits on LFV decays of vector mesons are derived in a model independent way. Assuming that a vector boson $M_{i}$ couples to $\mu^{\mp} e^{ \pm}$and $e^{\mp} e^{ \pm}$, the authors of Ref. 26 deduce some upper bounds on the LFV decays of vector mesons by a consideration of the experimental constraint on the process $\mu \rightarrow 3 e$. Under a similar assumption that a vector meson $M_{i}$ couples to $\mu^{\mp} e^{ \pm}$and nucleon-nucleon, Ref. 27 and Ref. 28 study the LFV decays of vector mesons by taking account of the experimental constraint on $\mu-e$ conversion.

In this paper, we investigate the LFV decays of vector mesons in unparticle physics by the consideration of constraint on $\mu-e$ conversion. In Section 2, we firstly provide a brief introduction to the unparticle physics and corresponding interaction Lagrangian in effective field theory. Then we derive the analytic results of the amplitude in detail. The numerical analysis and discussion are presented in Section, 3, and the conclusion is drawn in Section, 4

\section{Formalism}

In very high energy, as it is proposed by Georgi, 13114 the theory is composed of the SM fields and the fields of a theory with a nontrivial IR fixed point, which is called Banks-Zaks $(\mathcal{B Z})$ fields. 29 The two fields can interact by the exchange of particles with a large mass scale $M_{\mathcal{U}} \gg 1 T e V$. Below the scale $M_{\mathcal{U}}$, there are nonrenormalizable couplings involving both standard model fields and Banks-Zaks fields suppressed by powers of $M_{\mathcal{U}}$. The interaction between SM field and $\mathcal{B Z}$ field has the form:

$$
\frac{1}{M_{\mathcal{U}}^{d_{S M}+d_{\mathcal{B Z}}-4}} O_{S M} O_{\mathcal{B Z}}
$$

where $O_{S M}$ is an operator with a mass dimension $d_{S M}$ corresponding to SM fields and $O_{\mathcal{B Z}}$ is an operator with a mass dimension $d_{\mathcal{B Z}}$ corresponding to $\mathcal{B Z}$ fields. In 
effective field theory, below the scale $\Lambda_{\mathcal{U}}$, the $\mathcal{B Z}$ operators would match onto the unparticle operators, and Eq.(11) can be viewed as the interaction between SM field and unparticle field:

$$
\frac{C_{\mathcal{U}} \Lambda_{\mathcal{U}}^{d_{\mathcal{B}}-d_{\mathcal{U}}}}{M_{\mathcal{U}}^{d_{S M}+d_{\mathcal{B Z}}-4}} O_{S M} O_{\mathcal{U}}
$$

where $C_{\mathcal{U}}$ is a coefficient function, $d_{\mathcal{U}}$ denotes the scaling dimension of the unparticle operator $O_{\mathcal{U}}$.

For simplicity, it is convenient to define:

$$
\lambda=\frac{C_{\mathcal{U}} \Lambda_{\mathcal{U}}^{d_{\mathcal{Z}}}}{M_{\mathcal{U}}^{d_{S M}+d_{\mathcal{B Z}}-4}} .
$$

Then, in effective theory, the couplings of the scalar and vector unparticles to SM fermions (leptons or quarks) are generally given by the following effective operators:

$$
\begin{aligned}
& \frac{\lambda_{f f^{\prime}}^{S S}}{\Lambda_{\mathcal{U}}^{d_{\mathcal{U}}-1}} \bar{f} f^{\prime} O_{\mathcal{U}}, \frac{\lambda_{f f^{\prime}}^{S P}}{\Lambda_{\mathcal{U}}^{d_{\mathcal{U}}-1}} \bar{f} \gamma_{5} f^{\prime} O_{\mathcal{U}}, \frac{\lambda_{f f^{\prime}}^{S V}}{\Lambda_{\mathcal{U}}^{d_{\mathcal{U}}}} \bar{f} \gamma_{\mu} f^{\prime} \partial^{\mu} O_{\mathcal{U}}, \frac{\lambda_{f f^{\prime}}^{S A}}{\Lambda_{\mathcal{U}}^{d_{\mathcal{U}}}} \bar{f} \gamma_{\mu} \gamma_{5} f^{\prime} \partial^{\mu} O_{\mathcal{U}}, \\
& \frac{\lambda_{f f^{\prime}}^{V S}}{\Lambda_{\mathcal{U}}^{d_{\mathcal{U}}}} \bar{f} f^{\prime} \partial_{\mu} O_{\mathcal{U}}^{\mu}, \frac{\lambda_{f f^{\prime}}^{V P}}{\Lambda_{\mathcal{U}}^{d_{\mathcal{U}}}} \gamma_{5} f^{\prime} \partial_{\mu} O_{\mathcal{U}}^{\mu}, \frac{\lambda_{f f^{\prime}}^{V V}}{\Lambda_{\mathcal{U}}^{d_{\mathcal{U}}-1}} \bar{f} \gamma_{\mu} f^{\prime} O_{\mathcal{U}}^{\mu}, \frac{\lambda_{f f^{\prime}}^{V A}}{\Lambda_{\mathcal{U}}^{d_{\mathcal{U}}-1}} \bar{f} \gamma_{\mu} \gamma_{5} f^{\prime} O_{\mathcal{U}}^{\mu},
\end{aligned}
$$

where $\lambda_{f f^{\prime}}^{S, P, V, A}$ are dimensionless coefficients. $S, P, V$ and $A$ stand for scalar field, pseudo-scalar field, vector field and axial vector field, respectively. $f$ and $f^{\prime}$ denote SM fermions, $O_{\mathcal{U}}$ and $O_{\mathcal{U}}^{\mu}$ denote scalar and vector unparticle fields. The propagator of scalar unparticle field has the form $14 \mid 30$.

$$
\int e^{i P \cdot x} d^{4} x\langle 0| T\left[O_{\mathcal{U}}(x) O_{\mathcal{U}}(0)|0\rangle=i \frac{A_{d_{\mathcal{U}}}}{2 \sin \left(d_{\mathcal{U}} \pi\right)} \frac{1}{\left(-P^{2}-i \epsilon\right)^{d_{\mathcal{U}}-2}}\right.
$$

If the vector unparticle field is assumed to be transverse, the propagator can been written as:

$$
\int e^{i P \cdot x} d^{4} x\langle 0| T\left[O_{\mathcal{U}}^{\mu}(x) O_{\mathcal{U}}^{\nu}(0)|0\rangle=i \frac{A_{d_{\mathcal{U}}}}{2 \sin \left(d_{\mathcal{U}} \pi\right)} \frac{-g^{\mu \nu}+P^{\mu} P^{\nu}}{\left(-P^{2}-i \epsilon\right)^{2-d_{\mathcal{U}}}}\right.
$$

where $A_{d_{\mathcal{U}}}$ is defined by:

$$
A_{d_{\mathcal{U}}}=\frac{16 \pi^{5 / 2}}{(2 \pi)^{2 d_{\mathcal{U}}}} \frac{\Gamma\left(d_{\mathcal{U}}+1 / 2\right)}{\Gamma\left(d_{\mathcal{U}}-1\right) \Gamma\left(2 d_{\mathcal{U}}\right)}
$$

For vector mesons, only the vector current $\bar{f} \gamma_{\mu} f^{\prime}$ couples to vector mesons. The tree level Feynman diagram is presented in Fig[1] The amplitude for Fig:1 can be written as:

$$
\mathcal{M}_{Q}=\frac{\lambda_{b b}^{V V} \lambda_{e \mu}^{V V}}{\Lambda_{\mathcal{U}}^{2 d_{\mathcal{U}}-2}} \frac{A_{d_{\mathcal{U}}}}{2 \sin \left(d_{\mathcal{U}} \pi\right)} \bar{v}\left(p_{1}\right) \gamma_{\mu} u\left(p_{2}\right) \frac{g^{\mu \nu}-p^{\mu} p^{\nu}}{p^{2\left(2-d_{\mathcal{U}}\right)}} \bar{u}\left(p_{3}\right) \gamma_{\nu} v\left(p_{4}\right)
$$

In the quark picture, mesons are composed of a quark and an antiquark. We adopt a phenomenological model where the amplitude of hard process involving a s-wave meson can be described by the matrix elements of gauge-invariant nonlocal operators, 
Ke-Sheng Sun,etc.

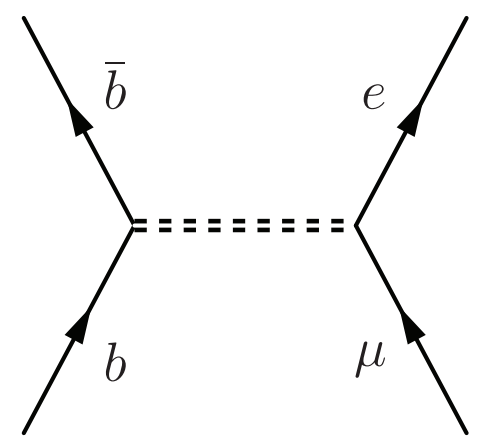

Fig. 1. The tree level diagram for LFV process $\Upsilon \rightarrow e \mu$, the double dashed line denotes the vector unparticle field.

which are sandwiched between the vacuum and the meson states. The distribution amplitude of vector meson $\Upsilon$ in leading-order is defined through the correlation function $31|32| 33$ :

$$
\begin{aligned}
\left\langle 0\left|\bar{b}_{1 \alpha}^{i}(y) b_{2 \beta}^{j}(x)\right| \Upsilon(p)\right\rangle= & \frac{\delta_{i j}}{4 N_{c}} \int_{0}^{1} d u e^{-i[u p y+(1-u) p x]}\left[f_{\Upsilon} m_{\Upsilon \notin} \phi_{\|}(u)\right. \\
& \left.+\frac{i}{2} \sigma^{\mu^{\prime} \nu^{\prime}} f_{\Upsilon}^{T}\left(\varepsilon_{\mu^{\prime}} p_{\nu^{\prime}}-\varepsilon_{\nu^{\prime}} p_{\mu^{\prime}}\right) \phi_{\perp}(u)\right]_{\beta \alpha},
\end{aligned}
$$

where $\mathrm{Nc}$ is the number of colors, $\varepsilon$ is the polarization vector, $f_{\Upsilon}$ and $f_{\Upsilon}^{T}$ are the decay constants, $\phi_{\|}$and $\phi_{\perp}$ are the leading-twist distribution functions corresponding to the longitudinally and transversely polarized meson, respectively. Since the leading-twist light-cone distribution amplitudes of meson are close to their asymptotic form, $\frac{34}{3}$ so we set $\phi_{\|}=\phi_{\perp}=\phi(u)=6 u(1-u)$.

Then, at hadron level, using Eq.(9), the amplitude is rewritten as

$$
\mathcal{M}_{H}=\frac{\lambda_{b b}^{V V} \lambda_{e \mu}^{V V}}{\Lambda_{\mathcal{U}}^{2 d_{\mathcal{U}}-2}} \frac{A_{d_{\mathcal{U}}} m_{\Upsilon} f_{\Upsilon}}{2 N_{c} \sin \left(d_{\mathcal{U}} \pi\right)} \frac{\varepsilon^{\nu}}{p^{2\left(2-d_{\mathcal{U}}\right)}} \bar{u}\left(p_{3}\right) \gamma_{\nu} v\left(p_{4}\right) .
$$

In the frame of center of mass, using the summation formula

$$
\sum_{\lambda= \pm 1,0} \varepsilon_{\lambda}^{\mu}(p) \varepsilon_{\lambda}^{* \nu}(p) \equiv-g^{\mu \nu}+\frac{p^{\mu} p^{\nu}}{m_{\Upsilon}^{2}}
$$

we get

$$
\left|\mathcal{M}_{H}\right|^{2}=\left|\frac{\lambda_{b b}^{V V} \lambda_{e \mu}^{V V}}{\Lambda_{\mathcal{U}}^{2 d_{\mathcal{U}}-2}} \frac{A_{d_{\mathcal{U}}} m_{\Upsilon} f_{\Upsilon}}{2 N_{c} \sin \left(d_{\mathcal{U}} \pi\right)}\right|^{2} \frac{4\left(m_{\Upsilon}^{2}-m_{e}^{2}-m_{\mu}^{2}\right)-16 m_{e} m_{\mu}}{m_{\Upsilon}^{4\left(2-d_{\mathcal{U}}\right)}} .
$$

Finally,we express the branching ratio of process $\Upsilon \rightarrow e \mu$ as

$$
B r(\Upsilon \rightarrow e \mu)=\frac{\sqrt{\left[m_{\Upsilon}^{2}-\left(m_{e}+m_{\mu}\right)^{2}\right]\left[m_{\Upsilon}^{2}-\left(m_{e}-m_{\mu}\right)^{2}\right]}}{16 \pi m_{\Upsilon}^{3} \Gamma \Upsilon} \times\left|\mathcal{M}_{H}\right|^{2},
$$

where $\Gamma_{\Upsilon}$ is the total decay width. The branching ratios for other LFV processes of vector mesons can be formulated in a similar way. 


\section{Numerical Analysis}

Taking account of the constraint on the LFV processes $\mu \rightarrow e \gamma$ and $\mu-e$ conversion in nuclei, we will study the LFV decay of $\Upsilon \rightarrow e \mu$ in unparticle physics firstly. Under the assumption of the unparticle couplings with the SM fermions in Eq.(4) are universal:

$$
\lambda_{f f^{\prime}}^{K K}=\left\{\begin{array}{r}
\lambda_{k}, f=f^{\prime} \\
\kappa \lambda_{k}, f \neq f^{\prime}
\end{array}\right.
$$

where $\kappa>1$ and $\mathrm{K}=\mathrm{S}, \mathrm{P}, \mathrm{V}, \mathrm{A}$ for scalar, pseudoscalar, vector and axial vector couplings respectively, the authors of Ref. 17 have investigated the LFV processes $\mu \rightarrow e \gamma$ and $\mu-e$ conversion in various nuclei in region of $1<d_{\mathcal{U}}<2,1 \mathrm{TeV}<\Lambda_{\mathcal{U}}<$ $100 \mathrm{TeV}$. It displays the constraint on dimension $d_{\mathcal{U}}$ deduced from experimental bound on $\mu-e$ conversion is more stringent. Therefore, we will study the LFV decays of vector mesons by a consideration of $\mu-e$ conversion in unparticle physics. The formula for the $\mu-e$ conversion rate with the pure vector coupling between SM fermions and unparticle is given by:

$$
\begin{aligned}
C R(\mu-e, N \text { ucleus })= & \frac{m_{\mu}^{5} \alpha^{3} Z_{\text {eff }}^{4} F_{p}^{2}}{2 \pi^{2} Z}\left[\lambda_{e \mu}^{V V} \lambda_{q q}^{V V} \frac{A_{d_{\mathcal{U}}}}{2 \sin \left(d_{\mathcal{U}} \pi\right)} \frac{1}{\Lambda_{\mathcal{U}}^{2}}\left(\frac{m_{\mu}^{2}}{\Lambda_{\mathcal{U}}^{2}}\right)^{d_{\mathcal{U}}-2}\right]^{2} \\
& \times\left|Z \sum_{q} G_{V}^{(q, p)}+N \sum_{q} G_{V}^{(q, n)}\right|^{2} \frac{1}{\Gamma_{\text {capt }}},
\end{aligned}
$$

where $\mathrm{Z}$ and $\mathrm{N}$ denote the proton and neutron numbers in a nucleus, $F_{p}$ is the nuclear form factor and $Z_{\text {eff }}$ is an effective atomic charge, $G_{V}^{(q, p)}$ and $G_{V}^{(q, n)}$ are nuclear matrix elements relevant to proton and neutron.

Here, as in Ref. 17, taking $\Lambda_{\mathcal{U}}=10 \mathrm{TeV}, \lambda_{b b}^{V V}=0.001$ and $\lambda_{e \mu}^{V V}=0.003$, we display $\operatorname{BR}(\Upsilon \rightarrow e \mu)$ versus $d_{\mathcal{U}}$ and $\mathrm{CR}(\mu-e, T i)$ versus $d_{\mathcal{U}}$ in region of $1 \leq d_{\mathcal{U}} \leq 4$ in Fig 2, where the solid line denotes the prediction of $\mathrm{CR}(\mu-e, T i)$, the dot line denotes the prediction of $\operatorname{BR}(\Upsilon \rightarrow e \mu)$. The horizontal lines correspond to $10^{-6}$ and $4.2 \times 10^{-12}$, which are the experimental sensitivity of LFV decays of vector mesons and the experimental bound on $\mu-e$ conversion rate respectively. The following numerical values are used $36|37| 38$ :

$$
\begin{aligned}
& m_{\Upsilon}=9.406 \mathrm{GeV}, f_{\Upsilon}=715 \mathrm{MeV}, \Gamma_{\Upsilon}=54 \mathrm{KeV}, \\
& F_{p}=0.54, Z_{\text {eff }}=17.6, \Gamma_{\text {capt }}=1.7 \times 10^{-18} .
\end{aligned}
$$

As we can see from Fig, 2 , the predictions of both $\operatorname{BR}(\Upsilon \rightarrow e \mu)$ and $\operatorname{CR}(\mu-$ $e, T i$ ) depend strongly on the scaling dimension $d_{\mathcal{U}}$. The value of dimension $d_{\mathcal{U}}$ is constrained to near 2 or more larger and the relevant prediction of $\operatorname{BR}(\Upsilon \rightarrow e \mu)$ is highly suppressed to reach the experimental sensitivity.

There is an interesting feature in Fig 2 that the prediction of $\operatorname{BR}(\Upsilon \rightarrow e \mu)$ is less than $\mathrm{CR}(\mu-e, T i)$ in region of $1 \leq d_{\mathcal{U}} \leq 3$, however, in region of $3 \leq$ $d_{\mathcal{U}} \leq 4$, the prediction of $\operatorname{BR}(\Upsilon \rightarrow e \mu)$ is larger than $\operatorname{CR}(\mu-e, T i)$. Considering 


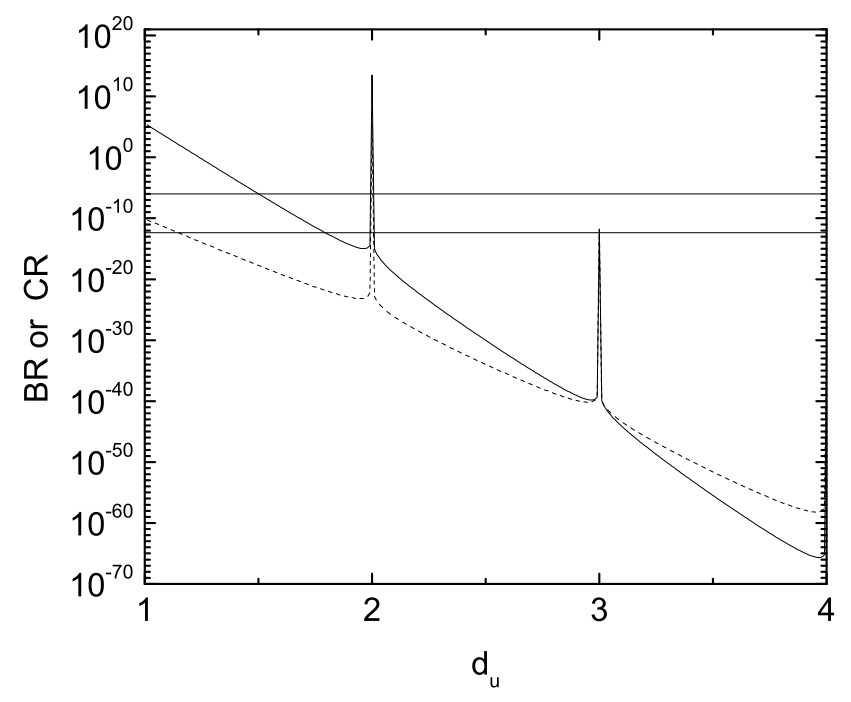

Fig. 2. The $\operatorname{BR}(\Upsilon \rightarrow e \mu)$ and $\operatorname{CR}(\mu-e, T i)$ vary as a function of $d_{\mathcal{U}}$, where the solid line denotes the prediction of $\mathrm{CR}(\mu-e, T i)$, the dot line denotes the prediction of $\mathrm{BR}(\Upsilon \rightarrow e \mu)$, the horizontal lines correspond to $10^{-6}$ and $4.2 \times 10^{-12}$, which are the experimental sensitivity of LFV decays of vector mesons and the experimental bound on $\mu-e$ conversion rate respectively. $\Lambda_{\mathcal{U}}=10 \mathrm{TeV}$, $\lambda_{b b}^{V V}=0.001$ and $\lambda_{e \mu}^{V V}=0.003$ are assumed.

the experimental bound on $\operatorname{CR}(\mu-e, T i)$ is $\mathcal{O}\left(10^{-12}\right)$, it is impossible to make the prediction of $\operatorname{BR}(\Upsilon \rightarrow e \mu)$ reach the experimental sensitivity by resetting the couplings $\left|\lambda_{b b}^{V V} \lambda_{e \mu}^{V V}\right|$ in region of $1 \leq d_{\mathcal{U}} \leq 3$. Nevertheless, in region of $3 \leq d_{\mathcal{U}} \leq 4$, by enlarging the couplings $\left|\lambda_{b b}^{V V} \lambda_{e \mu}^{V V}\right|$, it is available to get that not only the prediction of $\mathrm{CR}(\mu-e, T i)$ is compatible with the experimental bound, but also the prediction of $\operatorname{BR}(\Upsilon \rightarrow e \mu)$ is large enough to be detected in experiment at present or in near future. Therefore, we will investigate the LFV decays of vector mesons in region of $3 \leq d_{\mathcal{U}} \leq 4$. In addition, the constraint of $d_{\mathcal{U}} \geq 3$ is also supported in Ref. 39 and Ref. 40 by the consideration of unitarity. From unitarity, the gauge invariant primary vector operator $\mathcal{U}^{\mu}$ have $d_{V} \geq 3$, with $d_{V}=3$ if and only if the operator is a conserved current, $\partial_{\mu} \mathcal{U}^{\mu}=0$.

For the aim of enhancing the prediction of $\operatorname{BR}(\Upsilon \rightarrow e \mu)$ to be detectable in experiment, the value of the couplings $\left|\lambda_{b b}^{V V} \lambda_{e \mu}^{V V}\right|$ would be very large. However, we can also investigate the LFV decays of vector mesons in a way independent of the couplings $\left|\lambda_{b b}^{V V} \lambda_{e \mu}^{V V}\right|$. Considering the $\mu-e$ conversion in Ti nucleus, let us define the fraction $R(X)$ by:

$$
R(X)=\frac{B R(X \rightarrow e \mu)}{C R(\mu-e, T i)}
$$


where $X$ would be any vector mesons: $\rho, \omega, \phi, J / \Psi$ or $\Upsilon$. From Eq.(12), Eq.(13) and Eq.(15), we can see that coefficient $\lambda_{e \mu}^{V V}$ and mass scale $\Lambda_{\mathcal{U}}$ can be canceled out in $R(X)$. As for the unparticle couplings with the quarks are universal, i.e.,

$$
\lambda_{b b}^{V V}=\lambda_{s s}^{V V}=\lambda_{c c}^{V V} \simeq \frac{\lambda_{u u}^{V V}+\lambda_{d d}^{V V}}{\sqrt{2}} \simeq \frac{\lambda_{u u}^{V V}-\lambda_{d d}^{V V}}{\sqrt{2}}
$$

the couplings listed in Eq.(18) would also be canceled out in $R(X)$ due to the same value setting in both numerator and denominator in Eq.(17). Therefore, $R(X)$ would only be a function of scaling dimension $d_{\mathcal{U}}$. Using Eq.(12), Eq.(13) and Eq.(15), the relation between $R(X)$ and $d_{\mathcal{U}}$ can be shown in a simple form:

$$
R(X) \propto\left(\frac{m_{X}}{m_{\mu}}\right)^{4\left(d_{\mathcal{U}}-2\right)},
$$

where $\frac{m_{X}}{m_{\mu}}>1$ for different mesons. It is noteworthy that even if the unparticle couplings with the SM fermions are not universal, i.e., Eq.(18) is not feasible, the relation between $R(X)$ and $d_{\mathcal{U}}$ in Eq.(19) is still reliable.

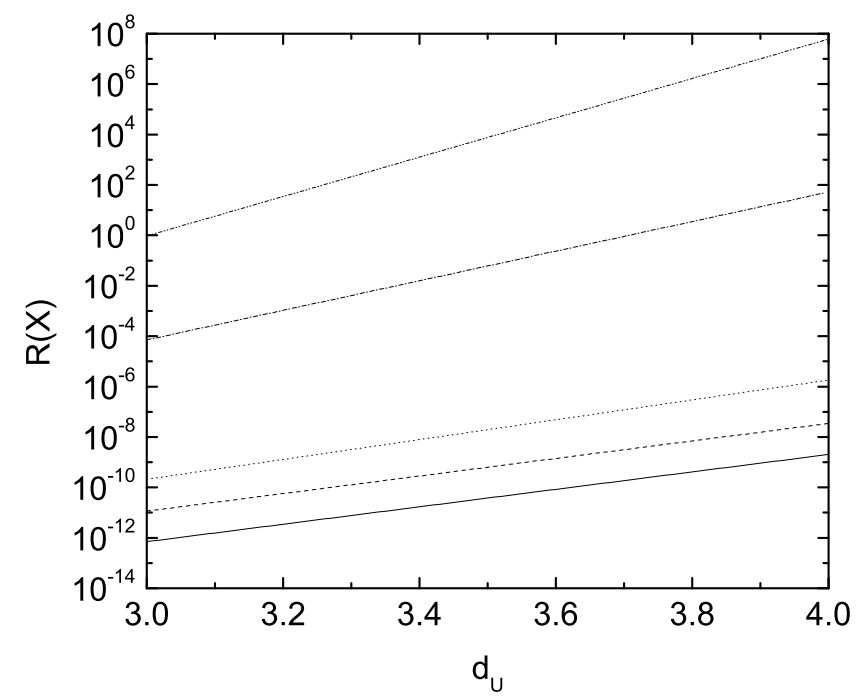

Fig. 3. The fraction $R(X)$ varies as a function of $d_{\mathcal{U}}$, where, from the bottom up, the lines stand for $R(\rho), R(\omega), R(\phi), R(J / \Psi)$ and $R(\Upsilon)$, respectively.

In Fig 3, we display the fraction $R(X)$ varies as a function of $d_{\mathcal{U}}$ in region of $3 \leq d_{\mathcal{U}} \leq 4$, where, from the bottom up, the lines stand for $R(\rho), R(\omega), R(\phi), R(J / \Psi)$ and $R(\Upsilon)$, respectively. The parameters relevant to different mesons are listed 
8 Ke-Sheng Sun,etc.

below $36|37| 38$ :

$$
\begin{aligned}
m_{\rho} & =775 \mathrm{MeV}, f_{\rho}=209 \mathrm{MeV}, \Gamma_{\rho}=149 \mathrm{MeV}, \\
m_{\omega} & =782 \mathrm{MeV}, f_{\omega}=195 \mathrm{MeV}, \Gamma_{\omega}=8.49 \mathrm{MeV}, \\
m_{\phi} & =1.019 \mathrm{GeV}, f_{\phi}=231 \mathrm{MeV}, \Gamma_{\phi}=4.2 \mathrm{MeV}, \\
m_{J / \Psi} & =3.096 \mathrm{GeV}, f_{J / \Psi}=405 \mathrm{MeV}, \Gamma_{J / \Psi}=92.9 \mathrm{KeV} .
\end{aligned}
$$

It displays in Fig 3 that $R(X)$ increases as $d_{\mathcal{U}}$ grows. For light flavor mesons, the fraction $R(X)$ is very small. However, for heavy flavor mesons, the fraction $R(X)$ is large and we can get a large $\operatorname{BR}(X \rightarrow e \mu)$ to be detectable in experiment and compatible with the constraint on $\mu-e$ conversion.

From Eq. (17) we can express the branching ratio of $X \rightarrow e \mu$ as:

$$
B R(X \rightarrow e \mu)=R(X) \times C R(\mu-e, T i) .
$$

Using Eq.(20), we give the predictions on branching ratios of LFV decays of vector mesons in Tab1 with $d_{\mathcal{U}}=3, d_{\mathcal{U}}=3.5$ and $d_{\mathcal{U}}=4$, where $\operatorname{CR}(\mu-e, T i) \leq$ $4.2 \times 10^{-12}$ is used. For light flavor mesons, the prediction of $\operatorname{BR}(X \rightarrow e \mu)$ is

Table 1. Predictions on branching ratios of LFV decays of vector mesons with $d_{\mathcal{U}}=3, d_{\mathcal{U}}=3.5$ and $d_{\mathcal{U}}=4$, where $\mathrm{CR}(\mu-e, T i) \leq 4.2 \times 10^{-12}$ is used.

\begin{tabular}{cccc}
\hline Decay & $d_{\mathcal{U}}=3$ & $d_{\mathcal{U}}=3.5$ & $d_{\mathcal{U}}=4$ \\
\hline$\rho \rightarrow e \mu$ & $2.8 \times 10^{-24}$ & $1.5 \times 10^{-22}$ & $8.1 \times 10^{-21}$ \\
$\omega \rightarrow e \mu$ & $4.6 \times 10^{-23}$ & $2.5 \times 10^{-21}$ & $1.3 \times 10^{-19}$ \\
$\phi \rightarrow e \mu$ & $8.4 \times 10^{-22}$ & $7.8 \times 10^{-20}$ & $7.2 \times 10^{-18}$ \\
$J / \Psi \rightarrow e \mu$ & $2.8 \times 10^{-16}$ & $2.4 \times 10^{-13}$ & $2.1 \times 10^{-10}$ \\
$\Upsilon \rightarrow e \mu$ & $3.8 \times 10^{-12}$ & $3.0 \times 10^{-8}$ & $2.4 \times 10^{-4}$ \\
\hline
\end{tabular}

much little, and it is impossible to observe the LFV processes of these mesons in experiment. For heavy flavor mesons, the large prediction of $\operatorname{BR}(X \rightarrow e \mu)$ is available for $d_{\mathcal{U}}$ near 4 . Especially, the prediction of $\operatorname{BR}(\Upsilon \rightarrow e \mu)$ is as large as $\mathcal{O}\left(10^{-4}\right)$, and it is very promising to be observed in experiment.

In literatures, several stringent limits on LFV decays of vector mesons are derived already. A summary table of experimental bounds and corresponding theoretical predictions is presented in Tab2 Assuming that a vector boson $M_{i}$ couples to $\mu^{\mp} e^{ \pm}$and $e^{\mp} e^{ \pm}$, the authors of Ref. 26 deduce some upper bounds on the LFV decay of mesons using the experimental constraint on the LFV process $\mu \rightarrow 3 e$. Under a similar assumption that a vector meson $M_{i}$ couples to $\mu^{\mp} e^{ \pm}$and nucleonnucleon, Ref. 27 and Ref. 28 study the LFV decays of vector mesons by taking account of the experimental constraint on $\mu-e$ conversion. From Tab 1 and Tab 2 it is easy to find that our predictions are compatible with those in literatures.

Finally, the predictions of LFV decays of vector mesons in both our article and Ref. 26, 27, 28 greatly depend on the experimental constraints on $\operatorname{BR}\left(l_{i} \rightarrow l_{j} \gamma\right)$, 
Table 2. The upper bounds on the branching ratios of vector meson decays in experiment and literatures.

\begin{tabular}{cccc}
\hline Decay & Exp & Ref. [26 & Ref. ? \\
\hline$\rho \rightarrow e \mu$ & - & $\leq 3.8 \times 10^{-20}$ & $\leq 3.5 \times 10^{-24}$ \\
$\omega \rightarrow e \mu$ & - & $\leq 8.1 \times 10^{-16}$ & $\leq 6.2 \times 10^{-27}$ \\
$\phi \rightarrow e \mu$ & $\leq 2.0 \times 10^{-6}$ & $\leq 4.0 \times 10^{-17}$ & $\leq 1.3 \times 10^{-21}$ \\
$J / \Psi \rightarrow e \mu$ & $<1.1 \times 10^{-6}$ & $\leq 4.0 \times 10^{-13}$ & $\leq 3.5 \times 10^{-13}$ \\
$\Upsilon \rightarrow e \mu$ & - & $\leq 2.0 \times 10^{-9}$ & $\leq 3.9 \times 10^{-6}$ \\
\hline
\end{tabular}

$\mathrm{BR}\left(l_{i} \rightarrow 3 l_{j}\right)$ and $\mathrm{CR}(\mu-e)$. Thus, more reliable predictions on LFV decays of vector mesons depend on the new data from the experiment. In the future, the expected sensitivities for BR $(\mu \rightarrow e \gamma)$ would be of order $10^{-13} \underline{42}$ For BR $(\tau \rightarrow e \gamma)$ and BR $(\tau \rightarrow \mu \gamma)$, it would be $10^{-9} \underline{43}$ For CR $(\mu-e, T i)$, it would be as low as $10^{-16} \sim 10^{-17}$ Then, the predictions of $\operatorname{BR}(X \rightarrow e \mu)$ for vector mesons would be more stringent.

\section{Conclusions}

Considering the constraint on $\mu-e$ conversion, we analyze the LFV decays of vector mesons in the framework of unparticle physics. In the scenario of the unparticle physics, the predictions of branching ratios of LFV decays of vector mesons depend strongly on the scale dimension $d_{\mathcal{U}}$. Supposing the unparticle couplings with the SM fermions are universal, the predictions of the branching ratios of the LFV decays of vector mesons can reach the detective sensitivity in experiment in region of $d_{\mathcal{U}} \geq$ 3 , while the prediction of $\mu-e$ conversion rate in Ti nucleus is compatible with the experimental upper limit. Although nonzero neutrino masses supported by the neutrino oscillation experiments imply the nonconservation of lepton flavors, it is very important to directly search the LFV processes of charged lepton sector in colliders running now. The LFV process $\Upsilon \rightarrow e \mu$ is very promising to be observed in experiment.

\section{Acknowledgements}

The work has been supported by the National Natural Science Foundation of China (NNSFC) with Grants No. 10975027.

\section{References}

1. Y. Fukuda et al. (Super Kamiokande Collab.), Phys. Rev. Lett81,1562(1998).

2. Q. R. Ahmad et al. (SNO Collab.), Phys. Rev. Lett37,071301(2001).

3. K. Eguchi et al. (Kamland Collab.), Phys. Rev. Lett90,021802(2003).

4. J.C. Pati and A. Salam, Phys. Rev. D10,275(1974).

5. H. Georgi and S. L. Glashow, Phys. Rev. Lett32,438(1974).

6. P. Langacker, Phys. Rep72,185(1981).

7. H. E. Haber and G. L. Kane, Phys. Rep117,75(1985). 
8. C.-H. Chang, T.-F. Feng, Eur. Phys. J.C12,137(2000).

9. K.-S. Sun, T.-F. Feng, T.-J. Gao, S.-M. Zhao, Nucl. Phys. B, DOI:10.1016/j.nuclphysb.2012.08.005.

10. R. N. Mohapatra and J. C. Pati, Phys. Rev. D11,566(1975).

11. R. N. Mohapatra and J. C. Pati, Phys. Rev. D11,2558(1975).

12. G. Senjanovic and R. N. Mohapatra, Phys. Rev. D12,1502(1975).

13. H. Georgi, Phys. Rev. Lett98,221601(2007).

14. H. Georgi, Phys. Lett. B650,275(2007).

15. T. M. Aliev, A. S. Cornell, N. Gaur, Phys. Lett. B657,27(2007).

16. D. Choudhury, D.K. Ghosh, Mamta, Phys. Lett. B658,148(2008).

17. G.-J. Ding, M.-L. Yan, Phys. Rev. D77,014005(2008).

18. C.-D. Lu, W. Wang, Y.-M. Wang, Phys. Rev. D76,077701(2007).

19. Wei.Zheng-Tao, Xu.Ye, Li.Xue-Qian, Eur. Phys. J. C62,593(2009).

20. E.O. Iltan,Eur. Phys. J. C56,105(2008).

21. E.O. Iltan, Mod. Phys. Lett. A23,3331(2008).

22. Zuo-Hong. Li,Ying. Li,Hong-Xia. Xu, Phys. Lett. B677,150(2009).

23. M. N. Achasov,et al. (SND Collaboration), Phys. Rev. D81,057102(2010).

24. M. Ablikim et al. (BES Collaboration), Phys. Lett. B598,172(2004).

25. W. Love et al. (CLEO Collaboration), Phys. Rev. Lett101,201601(2008).

26. S. Nussinov, R.D. Peccei, X.M. Zhang, Phys. Rev. D63,016003(2000).

27. T. Gutsche, J. Helo, S. Kovalenko, V.E. Lyubovitskij, Phys. Rev. D81,037702(2010).

28. T. Gutsche, J. Helo, S. Kovalenko, V.E. Lyubovitskij, Phys. Rev. D83,115015(2011).

29. T. Banks and A. Zaks, Nucl. Phys. B196,189(1982).

30. K. Cheung, W.Y. Keung and T. C. Yuan, Phys. Rev. Lett99,051803(2007).

31. T. Li, S.-M. Zhao, X.-Q. Li, Nucl. Phys. A828,125(2009).

32. P. Ball, V.M. Braun, Phys. Rev. D54,2182(1996).

33. T.-J. Gao, T.-F. Feng, X.-Q. Li, Z.-G. Si, S.-M. Zhao, Sci. China G53,1988(2010).

34. M. Beneke, G. Buchalla, M. Neubert, C. T. Sachrajda, Nucl. Phys. B591,313(2000).

35. S.-L. Chen, X.-G. He, X.-Q, Li, H.-C. Tsai, Z.-T. Wei,Eur. Phys. J.C59,899(2009).

36. Z.-Q. Zhang, Phys. Rev. D82,034036(2010).

37. Q. Wang,X.-H. Liu, Q. Zhao, hep-ph/1103.1095v1.

38. H.-W. Ke,X.-Q. Li,Z.-T. Wei, X. Liu, Phys. Rev. D82,034023(2010).

39. G. Mack,Commun. Math. Phys55(1977)1.

40. B. Grinstein, K.A. Intriligator, I.Z. Rothstein, Phys. Lett. B662,367(2008).

41. R. Kitano, M. Koike, and Y. Okada, Phys. Rev. D 66,096002(2002).

42. O. A. Kiselev [MEG Collaboration], Nucl. Instrum. Meth. A 604(2009)304.

43. M. Bona et al., arXiv:0709.0451 [hep-ex].

44. D. Glenzinski, AIP Conf. Proc. 1222 (2010) 383;Y. G. Cui et al. [COMET Collaboration], KEK-2009-10. 